\title{
Complete Response to Pembrolizumab in a Patient with Malignant Peripheral Nerve Sheath Tumor: The First Case Reported
}

\author{
Mehrdad Payandeh ${ }^{1}$, Masoud Sadeghi ${ }^{2,3^{*}}$, Edris Sadeghi ${ }^{2}$ \\ ${ }^{1}$ Department of Hematology and Medical Oncology, Kermanshah University of Medical Sciences, Kermanshah, Iran. \\ ${ }^{2}$ Medical Biology Research Center, Kermanshah University of Medical Sciences, Kermanshah, Iran. \\ ${ }^{3}$ Students Research Committee, Kermanshah University of Medical Sciences, Kermanshah, Iran.
}

\author{
ARTICLE INFO \\ Article history: \\ Received on: 03/07/2017 \\ Accepted on: 10/09/2017 \\ Available online: 30/10/2017 \\ Key words: \\ Malignant peripheral nerve \\ sheath tumor, pembrolizumab, \\ procarbazine.
}

\begin{abstract}
Pembrolizumab and procarbazine have been developed as anticancer agents. This study aimed to evaluate the efficacy of the combination of pembrolizumab plus procarbazine in a case with malignant peripheral nerve sheath tumor (MPNST (for the first time. A 48-year-old man referred to the Clinic of Oncology with complaints of constant abdominal pain for a week that the pathology diagnosis showed MPNST. The pathologist reported spindle cell sarcoma (probably dedifferentiated liposarcoma) with a significant PD-L1 expression-tumor proportion score (TPS): $90 \%$. The patient was treated with six courses bi-weekly pembrolizumab combined with procarbazine that after this treatment, abdominopelvic CT scan showed that his lesions completely were resolved. In conclusion, the combination of pembrolizumab with procarbazinecan be a new treatment in the patients with MPNST that in the future studies, the clinicians can check PD-L1 in these patients for better therapeutic aims.
\end{abstract}

\section{INTRODUCTION}

Malignant peripheral nerve sheath tumors (MPNSTs) are aggressive sarcomas of soft tissue distinguished with high risk of local recurrence and distant metastasis (Bradford and Kim, 2015; Farid et al., 2014) that pose tremendous challenges to effective (Farid et al., 2014). Pembrolizumab is a monoclonal antibody that binds to the PD-1 receptor and blocks interaction with its ligands (PD-L1 and PD-L2). This binding activates Tcell-mediated immune responses against cancer cells (Raedler, 2015). Procarbazine hydrochloride $(\mathrm{HCl})$ is an oral alkylating agent similar to dacarbazine and hexamethylamine. This drug was synthesized for the first time in the late 1950s during a search for a new monoamine oxidase inhibitor, but was soon

* Corresponding Author

Masoud Sadeghi, Kermanshah University of Medical Sciences, Kermanshah, Iran Email: Sadeghi_mbrc@yahoo.com developed as an antitumor agent (Friedman, 2001). The present study evaluated the efficacy of combination of pembrolizumab plus procarbazine $\mathrm{HCl}$ in a case with MPNST for the first time.

\section{Case Report}

On January 2016, a 48-year-old man referred to the Clinic of Oncology with complaints of constant abdominal pain for a week. The abdominopelvic CT scan showed retroperitoneal masses that the pathology diagnosis showed MPNST and he referred again to the clinic (Figure 1). Microscopically, a specimen of the patient contained infiltrates of a mesenchymal proliferation composed of spindled tumor cells.

In immunohistochemistry (IHC) evaluation, MDM2 protein (scattered), CD54, CD56, S100, SMA were positive, whereas desmin, B-catenin, synaptophysin, CD34, and DOG1 were negative. Also, Ki67 was positive in $10 \%$ of tumoral cells and CD117 (c-Kit) was weakly positive (1-2\% of tumoral cells). 


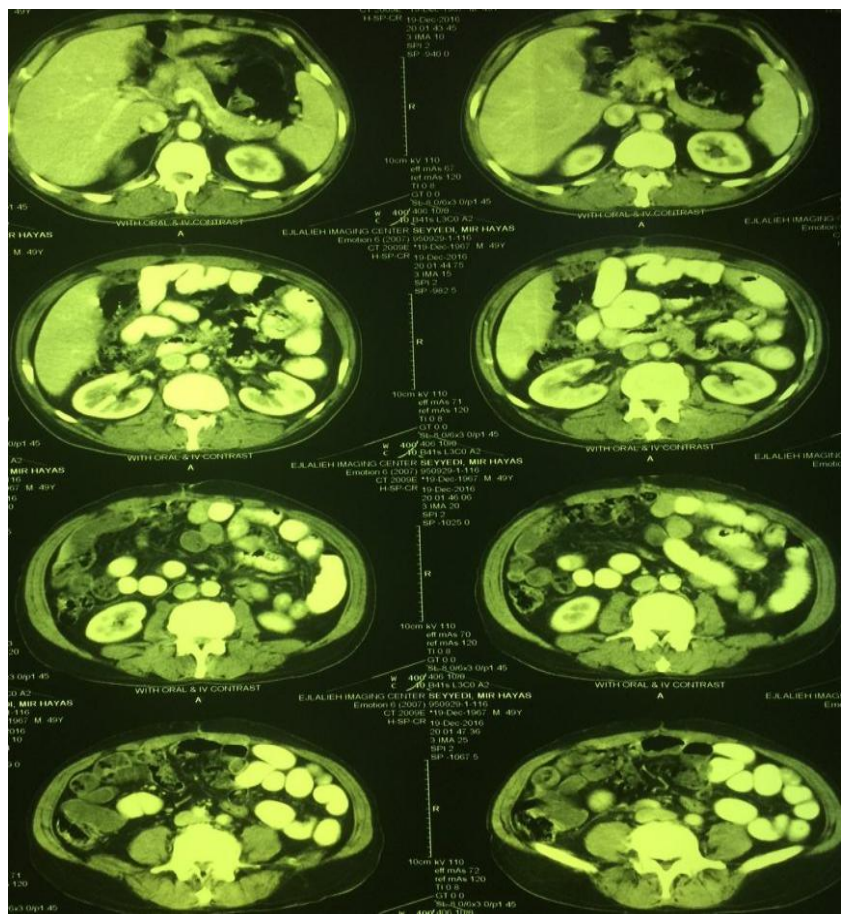

Fig. 1: Malignant peripheral nerve sheath tumor

By surgery a limited debulking was done, and he treated with six courses combination of doxorubicin $\left(100 \mathrm{mg} / \mathrm{m}^{2}\right.$ per day) and ifosfamide ( $3 \mathrm{~g} / \mathrm{m}^{2}$ for 3 days). In follow-up, there were the bulk of mass lesions in the CT scan (Figure 2).

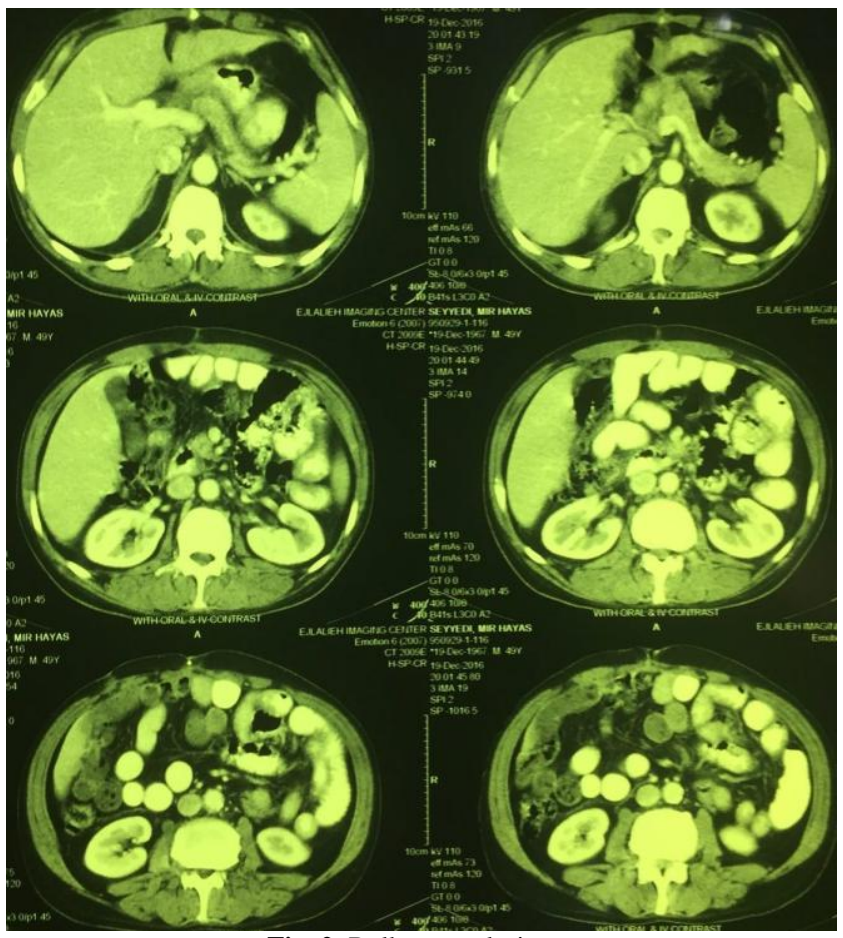

Fig. 2: Bulky mass lesions.

Therefore, the patient was treated with imatinib $400 \mathrm{mg}$ per day. After 3 months from this process, he referred again with generalized abdominal pain. On September 2016, the CT scan showed a mesenteric solid mass in the dimensions of $30 \times 44 \mathrm{~mm}$ in a position inferior of the stomach with an adhesion to the stomach wall and was suggestive of previous tumor recurrence in this location (Figure 3).

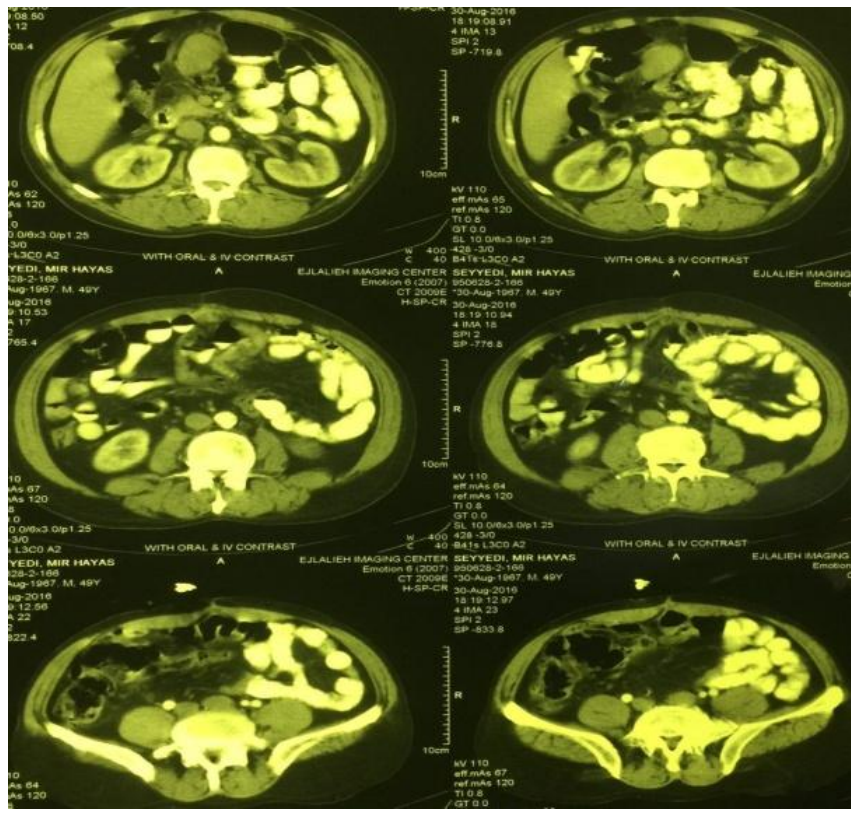

Fig. 3: Mesenteric solid mass in the inferior of the stomach

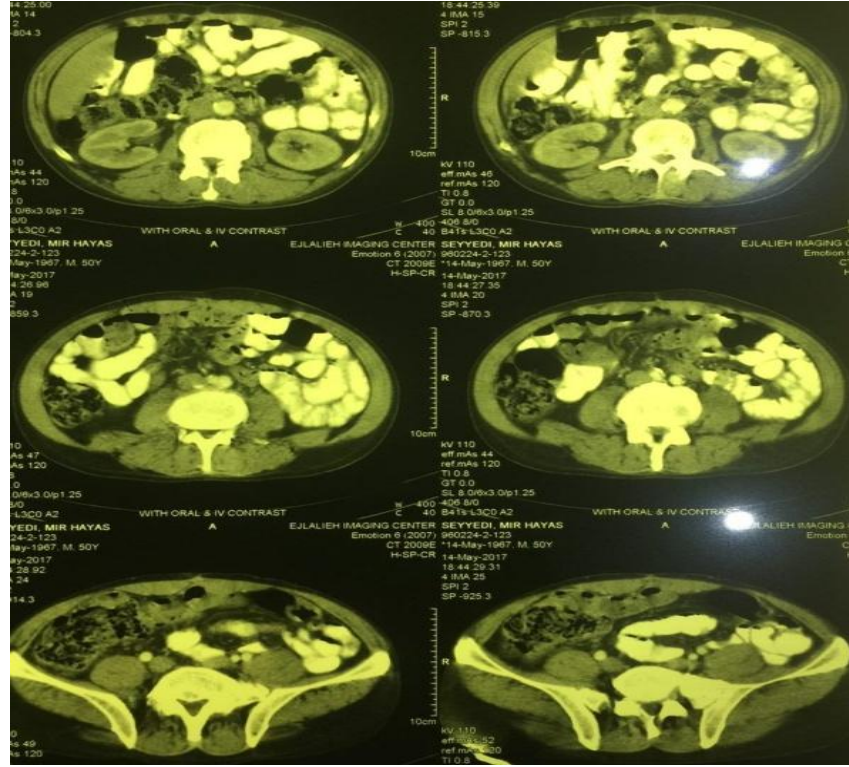

Fig. 4: Lesions completely were resolved after pembrolizumab therapy.

The patient was admitted for three surgical consults and abdominal mass debulking resection. In this line, he was treated with eribulin $\left(1.4 \mathrm{mg} / \mathrm{m}^{2}\right.$ IV infused for $2-5 \mathrm{~min}$ on days 1 and 8)for six cycles. The new specimen was contained from more than 100 viable tumor cells and was suitable for PD-L1 test. The IHC was carried out by using the DAKO $22 \mathrm{C} 3$ pharm DX kit on an Autostainer Link 48 platform for a PD-L1 test that suitable negative controls were included. The pathologist reported spindle cell sarcoma (probably dedifferentiated liposarcoma) with 
significant PD-L1 expression-tumor proportion score (TPS): $90 \%$. The patient was treated with six courses biweekly Keytruda (pembrolizumab) (200mg every 3 weeks) combined with procarbazine hydrochloride $(\mathrm{HCl})(50 \mathrm{mg} / \mathrm{m} 2$ twice a day). In new abdominopelvic CT scan, lesions completely were resolved (Figure 4) and he was followed during last 3 months. The patient is alive on Jun 2017.

\section{DISCUSSION}

This study showed that for the first time, pembrolizumab combined with procarbazine therapy had a complete response in MPNST patient.

Pembrolizumab was approved by the Unites States Food and Drug Administration (FDA) as the first anti-PD-1 antibody in the treatment of unresectable or metastatic melanoma patients with disease progression following ipilimumab that if mutant BRAF as a BRAF inhibitor (Khoja et al., 2015). This drug is a high-affinity, highly selective monoclonal antibody against PD-L1 that has shown important clinical activity in multiple tumor types (Khoja et al., 2015). A phase II clinical trial (SARC028)is doing on the efficacy of pembrolizumab in patients with MPNST (Guren TK,2016)that these patients will receive pembrolizumab therapy for up to 10 courses. A few trials are doing to evaluate the best use of pembrolizumab alone in melanoma, non-small cell lung cancer, and other tumor types (Khoja et al., 2015), as low-dose pembrolizumab (cumulatively only $6 \mathrm{mg} / \mathrm{kg}$ at interim assessment) was used to treat Hodgkin's lymphoma that resulted in an excellent response with the lowest adverse events and cost (Kwong et al., 2017).

Procarbazine $\mathrm{HCl}$ has been introduced as an oral alkylating agent with activity against lymphoma (Chaar et al., 2006). Insufficient results with standard agents have caused an interesting on developed targeted therapeutics of MPNST that this interest and desire build a large number of molecular data surrounding MPNST pathogenesis (Farid et al., 2014). Response rate in combination of doxorubicin plus ifosfamide (the most active agents) in unselected soft tissue sarcomas was approximately $25 \%$ that this response in MPNST was $21 \%$ (Kroep et al., 2011). The present study reported that the combination of these two drugs didn't have good response in MPNST patient. Recently, there are renew interests in procarbazine combinations with other chemotherapeutic factors, specifically in the treatment of Hodgkin's lymphoma, gliomas and with a less extent in nonHodgkin's lymphoma and primary central nervous system lymphoma (Armand et al., 2007). A case reported (Payandeh et $a l$. , 2015) showed maintenance therapy with procarbazine and chlorambucil is a new option for the patient with Hodgkin's lymphoma that reduced lesions of liver and lung and had a complete response in the patient. To conclude, the combination of pembrolizumab with procarbazinecan be a new treatment in the patients with MPNST that in the future studies, the clinicians can checkPD-L1 in these patients for better therapeutic aims.
Financial support or sponsorship: None.

Conflict of Interest: There is no conflict of interest.

\section{INFORMED CONSENT}

The informed consent was signed by the patient for reporting this case.

\section{REFERENCES}

Armand JP, Ribrag V, Harrousseau JL, Abrey L. Reappraisal of the use of procarbazine in the treatment of lymphomas and brain tumors. Ther Clin Risk Manag. 2007;3 (2):213-24.

Bradford D, Kim A. Current treatment options for malignant peripheral nerve sheath tumors. Curr Treat Options Oncol. 2015; 16 (3): 328.

Chaar BT, Salem P, Petruska PJ. Procarbazine for nonHodgkin's lymphoma. Leuk Lymphoma. 2006; 47 (4):637-40.

Farid M, Demicco EG, Garcia R, Ahn L, Merola PR, Cioffi A, et al. Malignant peripheral nerve sheath tumors. Oncologist. 2014;19 (2): 193-201

Friedman HS. In: Alkylating agents in cancer chemotherapy and biotherapy. 4th edition. Chabner BA, Longo DL, editors. Philadelphia: Lippincott Williams \& Wilkins; 2001. p. 415.

Guren TK. A Study of Pembrolizumab in Patients With Malignant Peripheral Nerve Sheath Tumor (MPNST), Not Eligible for Curative Surgery. 2016. Available: https://clinicaltrials.gov

Khoja L, Butler MO, Kang SP, Ebbinghaus S, Joshua AM. Pembrolizumab. J Immunother Cancer. 2015;3:36.

Kroep JR, Ouali M, Gelderblom H, Le Cesne A, Dekker TJ, Van Glabbeke M, et al. First-line chemotherapy for malignant peripheral nerve sheath tumor (MPNST) versus other histological soft tissue sarcoma subtypes and as a prognostic factor for MPNST: An EORTC soft tissue and bone sarcoma group study. Ann Oncol. 2011; 22 (1): 207-14.

Kwong YL, Lopes D, Khong PL. Low-dose pembrolizumab induced remission in patients with refractory classical Hodgkin lymphoma. Br J Haematol. 2017;176 (1):131-2.

Payandeh M, Sadeghi M, Sadeghi E. Maintenance Therapy with Procarbazin and Chlorambucil without Autologous Stem Cell Transplantation as a New Option for Patient with Hodgkin Lymphoma. Sch J App Med Sci. 2015;3 (1E):363-6.

Raedler LA. Keytruda (Pembrolizumab): First PD-1 Inhibitor Approved for Previously Treated Unresectable or Metastatic Melanoma. Am Health Drug Benefits. 2015; 8 (Spec Feature):96-100.

\section{How to cite this article:}

Payandeh M, Sadeghi M, Sadeghi E. Complete Response to Pembrolizumab in a Patient with Malignant Peripheral Nerve Sheath Tumor: The First Case Reported. J App Pharm Sci, 2017; 7 (10): 182-184. 